CTP-TAMU-12/96

$\mathrm{IC} / 96 / 54$

hep-th/9604058

\title{
Liouville and Toda Solitons in M-theory
}

\author{
H. Lü ${ }^{\dagger}$, C.N. Pope ${ }^{\dagger}$ \\ Center for Theoretical Physics, Texas A\&M University, College Station, Texas 77843 \\ K.W. Xu \\ International Center for Theoretical Physics, Trieste, Italy \\ and \\ Institute of Modern Physics, Nanchang University, Nanchang, China
}

\begin{abstract}
$\underline{\text { ABSTRACT }}$
We study the general form of the equations for isotropic single-scalar, multi-scalar and dyonic $p$-branes in superstring theory and M-theory, and show that they can be cast into the form of Liouville, Toda (or Toda-like) equations. The general solutions describe nonextremal isotropic $p$-branes, reducing to the previously-known extremal solutions in limiting cases. In the non-extremal case, the dilatonic scalar fields are finite at the outer event horizon.
\end{abstract}

\footnotetext{
${ }^{\dagger}$ Research supported in part by DOE Grant DE-FG05-91-ER40633
} 


\section{Introduction}

Solitonic extended-object solutions in the low energy effective field theories of the superstring and M-theory have been extensively studied. Until recently the principal focus has been on solutions that preserve some fraction of the supersymmetry, since these are expected to be associated with BPS saturated states in the superstring or M-theory. The purpose of this paper is to re-examine the general equations of motion describing the $p$ brane solutions. In particular we shall focus on isotropic $p$-brane solutions [1-11], for which the D-dimensional metric takes the form

$$
d s^{2}=e^{2 A} d x^{\mu} d x^{\nu} \eta_{\mu \nu}+e^{2 B} d y^{m} d y^{m}
$$

where $x^{\mu}$ are the coordinates of the $d$-dimensional world volume of the $p$-brane, $y^{m}$ are the coordinates of the $(D-d)$-dimensional transverse space, and $A$ and $B$ are functions of $r=\sqrt{y^{m} y^{m}}$ only.

The usual supersymmetric solutions are obtained as a subset of the most general solutions, by imposing a relation on the functions $A$ and $B$, namely $d A+\tilde{d} B=0$, where $\tilde{d}=D-d-2$. In this paper, we shall construct the most general solutions for isotropic $p$-brane solitons, in which neither this, nor any other condition, is imposed. Such a general solution has an additional integration constant, which implies that the mass per unit $p$-volume becomes independent of the charges, giving rise to a non-extremal $p$-brane. The dilatonic scalar fields are finite at the outer event horizon. We obtain such solutions for single-scalar, dyonic and multi-scalar $p$-branes. The equations of motion for the single-scalar $p$-branes can be cast into the form of the Liouville equation. The dyonic $p$-brane equations in general take the form of Toda-like equations for two independent variables. For two special cases for the dilaton dependence, these equations reduce to completely-solvable systems; in one case a pair of Liouville equations, and in the other, the $S U(3)$ Toda equations. In the case of multi-scalar $p$-branes involving $N$ scalars and $N$ field strengths, the equations are in general Toda-like, butu become become $N$ independent Liouville equations in the important subset of cases where the extremal limit is supersymmetric. All these equations can be derived from Hamiltonians, which are non-vanishing for generic solutions, but vanishes in the extremal limit. It should be remarked that for $d \geq 2$ these general non-extremal solutions are distinct from the usual black $p$-branes, whose world volume symmetry is broken to $R$ times the Euclidean group $E^{d-1}$ [12], in that here the full Poincare symmetry of the $d$-dimensional world volume is preserved. We shall carry out the construction of the general solutions in the subsequent three sections. 


\section{General electric and magnetic single-scalar solutions}

Let us begin by considering a $D$-dimensional bosonic Lagrangian of the form

$$
\mathcal{L}=e R-\frac{1}{2}(\partial \phi)^{2}-\frac{1}{2 n !} e^{a \phi} F_{n}^{2}
$$

where $F_{n}$ is an $n$ 'th rank antisymmetric tensor field strength, and $a$ is a constant, which can be conveniently parameterised as

$$
a^{2}=\Delta-\frac{2 d \tilde{d}}{D-2} .
$$

The Lagrangian describes a subset of the fields in the low-energy effective field theory of a string. As is well known, the equations of motion admit isotropic $p$-brane solutions whose world volume dimension $d$ is equal to $d=n-1$ for elementary solutions (with electric charge), or $d=D-n-1$ for solitonic solutions (with magnetic charge). The $D$-dimensional metric takes the form (11), and the field strength for elementary or solitonic solutions is given by

$$
F_{m \mu_{1} \cdots \mu_{n-1}}=\epsilon_{\mu_{1} \cdots \mu_{n-1}} \partial_{m} e^{C}, \quad \text { or } \quad F_{m_{1} \cdots m_{n}}=\lambda \epsilon_{m_{1} \cdots m_{n} p} \frac{y^{p}}{r^{n+1}} .
$$

The equations for $A, B, C$ and $\phi$ that follow by substituting the above ansätze into the equations of motion obtained from (2) are usually solved by making certain simplifying assumptions that are motivated by requiring that some fraction of the supersymmetry be preserved. In this paper, we shall obtain general solutions, in which no such further assumptions are made. To do this, we begin by re-expressing $A, B$ and $\phi$ in terms of three new quantities $X, Y$ and $\Phi$, defined by

$$
X \equiv d A+\tilde{d} B, \quad Y \equiv A+\frac{\epsilon \tilde{d}}{a(D-2)} \phi, \quad \Phi \equiv \epsilon a \phi-2 d A
$$

Defining the new radial variable $\rho=r^{-\tilde{d}}$, the equations of motion become

$$
\begin{aligned}
& X^{\prime \prime}+X^{\prime 2}-\frac{1}{\rho} X^{\prime}=0, \quad Y^{\prime \prime}+X^{\prime} Y^{\prime}=0, \\
& \Phi^{\prime \prime}+X^{\prime} \Phi^{\prime}=-\frac{\Delta \lambda^{2}}{2 \tilde{d}^{2}} e^{-\Phi-2 X}, \\
& \frac{a^{2}(D-2) d}{\Delta} Y^{\prime 2}-(\tilde{d}+1)\left(X^{\prime 2}-\frac{2}{\rho} X^{\prime}\right)+\frac{\tilde{d}}{2 \Delta} \Phi^{\prime 2}=\frac{\lambda^{2}}{2 \tilde{d}} e^{-\Phi-2 X},
\end{aligned}
$$

where a prime denotes a derivative with respect to $\rho$. In the case of elementary solutions, the charge parameter $\lambda$ in ([7) and (8) arises as the integration constant for the equation of motion for the function $C$. The first two equations (6) are easily be solved, giving $e^{X}=1-k^{2} \rho^{2}$ and $Y=-(\mu / k) \operatorname{arctanh}(k \rho)$, where $k$ and $\mu$ are arbitrary constants. Note 
that in the usual $p$-brane solutions, the functions $X$ and $Y$ are both taken to be zero, corresponding to $k=0=\mu$. In order to solve the remaining equations for $\Phi$, it is convenient to make the coordinate transformation

$$
k \rho=\tanh (k \xi)
$$

which has the property that $e^{X} \partial / \partial \rho=\partial / \partial \xi$. The equations (77) and (8) then reduce to

$$
\begin{aligned}
& \ddot{\Phi}=-\frac{\Delta \lambda^{2}}{2 \tilde{d}^{2}} e^{-\Phi} \\
& \frac{\Delta \lambda^{2}}{2 \tilde{d}} e^{-\Phi}-\frac{1}{2} \tilde{d} \dot{\Phi}^{2}=a^{2} d(D-2) \mu^{2}-4 \Delta(\tilde{d}+1) k^{2}
\end{aligned}
$$

where the dot denotes the derivative with respect to the redefined radial variable $\xi$. Thus $\Phi$ satisfies the Liouville equation (10), subject to the first integral (11). This can be reexpressed in the form of the Hamiltonian

$$
H \equiv \frac{1}{2} p^{2}-\frac{\Delta \lambda^{2}}{2 \tilde{d}^{2}} e^{-\Phi}=\frac{4 \Delta}{\tilde{d}}(\tilde{d}+1) k^{2}-a^{2} \frac{d}{\tilde{d}}(D-2) \mu^{2},
$$

where we have introduced the momentum $p$ conjugate to $\Phi$ as an auxiliary variable. Hamilton's equations $\partial H / \partial p=\dot{\Phi}$ and $\partial H / \partial \Phi=-\dot{p}$ then give (10), and the right-hand side of (12) gives the value of the energy, which is of course conserved, in terms of the arbitrary constants $k$ and $\mu$. Thus we find that the general solution is given by

$$
\begin{aligned}
& Y=-\mu \xi, \quad e^{-\frac{1}{2} X}=\cosh (k \xi), \\
& e^{\frac{1}{2} \Phi}=\frac{\lambda \sqrt{\Delta}}{2 \tilde{d} \beta} \sinh (\beta \xi+\alpha)
\end{aligned}
$$

where $\alpha, \mu, k$ and $\lambda$ are free parameters, and $\tilde{d} \beta^{2}=2(\tilde{d}+1) \Delta k^{2}-\frac{1}{2} a^{2} d(D-2) \mu^{2}$. The functions $A, B$ and $\phi$ are therefore given by

$$
\begin{aligned}
e^{-\frac{(D-2) \Delta}{2 \tilde{d}} A} & =\frac{\lambda \sqrt{\Delta}}{2 \tilde{d} \beta} \sinh (\beta \xi+\alpha) e^{a^{2}(D-2) \mu \xi /(2 \tilde{d})} \\
e^{\frac{(D-2) \Delta}{2 d} B} & =\frac{\lambda \sqrt{\Delta}}{2 \tilde{d} \beta} \sinh (\beta \xi+\alpha) e^{a^{2}(D-2) \mu \xi /(2 \tilde{d})}(\cosh (k \xi))^{-\frac{(D-2) \Delta}{d \tilde{d}}} \\
e^{\frac{\epsilon \Delta}{2 a} \phi} & =\frac{\lambda}{2 \tilde{d} \beta} \sinh (\beta \xi+\alpha) e^{-d \mu \xi}
\end{aligned}
$$

We shall choose $\alpha$ so that $\sinh \alpha=2 \tilde{d} \beta /(\lambda \sqrt{\Delta})$, implying that $A, B$ and $\phi$ go to zero at $r=\infty$ (i.e. at $\xi=0$ ). In particular, this means that the metric (1) approaches $D$ dimensional Minkowski spacetime at infinity. This asymptotically Minkowskian solution has a total of three independent free parameters, namely $\lambda, k$ and $\mu$. The metric is singular 
at $\xi=\infty$, corresponding to $r^{\tilde{d}}=k$. In order to interpret this as an outer event horizon, we must require that there be no curvature singularity at this location. It is also appropriate to impose the requirement that the dilaton $\phi$ remain finite at the outer event horizon, which implies that $\beta=\mu d$. Thus we have $\mu=2 k \sqrt{(\tilde{d}+1) /(d(D-2))}$. In fact this latter requirement also ensures the absence of curvature singularities at the outer horizon. The mass per unit $p$-volume and the charge are given in terms of the two remaining free parameters by

$$
m=\frac{\lambda}{2 \sqrt{\Delta}} \cosh \alpha+\frac{a^{2}(D-2) \mu}{2 \Delta}, \quad Q=\frac{\lambda}{4} .
$$

The standard extremal solution corresponds to taking $k=0$, implying that $X=Y=0$ and $e^{\frac{1}{2} \Phi}=1+\lambda \sqrt{\Delta} /(2 \tilde{d}) r^{-\tilde{d}}$, and also that $\mu=0$ and $\alpha=0$, giving rise to the standard mass/charge relation $m=2 Q / \sqrt{\Delta}$. If $k$ is instead positive, the mass exceeds this minimum value, and the solution describes a non-extremal black $p$-brane.

\section{General multi-scalar solutions}

We now turn to the discussion of multi-scalar $p$-brane solutions, where a set of $N$ field strengths carry independent charges. The relevant part of the supergravity Lagrangian in this case is given by

$$
e^{-1} \mathcal{L}=R-\frac{1}{2}(\partial \vec{\phi})^{2}-\frac{1}{2 n !} \sum_{i=1}^{N} e^{\vec{a}_{i} \cdot \vec{\phi}} F_{i}^{2},
$$

where $\vec{\phi}$ denotes a set of $N$ dilatonic scalar fields, and $F_{i}$ denotes a set of $N$ field strengths of rank $n$, with associated dilaton vectors $\vec{a}_{i}$. We shall consider only solutions that are purely elementary or purely solitonic, with each field strength given by an ansatz of the form (4).

First, we substitute the ansätze (1) and (1) into the equations of motion coming from (17). As in the single-scalar case in the previous section, it is advantageous to introduce new variables in order to diagonalise the equations. Accordingly, we begin by defining

$$
\begin{aligned}
X & =d A+\tilde{d} B, \quad Y=A+\frac{\epsilon \tilde{d}}{D-2} \sum_{i, j}\left(M^{-1}\right)_{i j} \varphi_{i}, \\
\Phi_{i} & =\epsilon \varphi_{i}-2 d A
\end{aligned}
$$

where $M_{i j} \equiv \vec{a}_{i} \cdot \vec{a}_{j}$, and $\varphi_{i} \equiv \vec{a}_{i} \cdot \vec{\phi}$. The form of the matrix $M_{i j}$ is determined by the details of the supergravity theory under consideration, and the particular subset of $N$ field strengths that are non-vanishing in the solution. One particularly important case that can arise is when 11]

$$
M_{i j}=4 \delta_{i j}-\frac{2 d \tilde{d}}{D-2}
$$


Note that the number $N$ of field strengths whose dilaton vectors $\vec{a}_{i}$ satisfy the above equation depends on the specific supergravity theory, and was discussed in the context of maximal supergravities in [10]. The standard multi-scalar solution that arises when $M_{i j}$ is given by (19) is supersymmetric, and if the $N$ independent charges $\lambda_{i}$ are set equal, the solution reduces to a single-scalar supersymmetric $p$-brane with $\Delta=4 / N$ [10, 11]. When $M_{i j}$ takes the form (19), we find that the equations of motion for $X, Y$ and $\Phi_{i}$ defined in (18) become

$$
\begin{aligned}
& X^{\prime \prime}+X^{\prime 2}-\frac{1}{\rho} X^{\prime}=0, \quad Y^{\prime \prime}+X^{\prime} Y^{\prime}=0, \\
& \ddot{\Phi}_{i}=-\frac{2 \lambda_{i}^{2}}{\tilde{d}^{2}} e^{-\Phi_{i}}, \\
& \sum_{i=1}^{N}\left(\frac{2 \lambda_{i}^{2}}{\tilde{d}} e^{-\Phi_{i}}-\frac{1}{2} \tilde{d} \dot{\Phi}_{i}^{2}\right)=-16(\tilde{d}+1) k^{2}+2 d(2(D-2)-d \tilde{d} N) \mu^{2},
\end{aligned}
$$

where a dot again means a derivative with respect to $\xi$, defined by (9). As in the singlescalar case, the solutions for $X$ and $Y$ are given by (13). The remaining equations (21) are $N$ independent Liouville equations for the functions $\Phi_{i}$, subject to the single first-integral constraint (22). This can be re-expressed in terms of the Hamiltonian

$$
H \equiv \sum_{i=1}^{N}\left(\frac{1}{2} p_{i}^{2}-\frac{2 \lambda_{i}^{2}}{\tilde{d}^{2}} e^{-\Phi_{i}}\right)=\frac{16(\tilde{d}+1)}{\tilde{d}} k^{2}-\frac{2 d}{\tilde{d}}(2(D-2)-d \tilde{d} N) \mu^{2},
$$

where $p_{i}$ is the momentum conjugate to $\Phi_{i}$. Hamilton's equations give rise to (21). Note that the equations for $\Phi_{i}$ are diagonalised into a set of $N$ Liouville equations (21) because of the choice of the dot products $M_{i j}$ of the dilaton vectors, given by (19). For other choices of $M_{i j}$, the equations will not be diagonalised, but will instead have a structure of the general form of the Toda equations. However, the specific possibilities for $M_{i j}$ that are allowed by supergravity theories do not give the precise coefficients that would correspond to any Toda equations for any Lie algebra.

The solutions of the Liouville equations (21) for $\Phi_{i}$ imply that

$$
e^{\frac{1}{2} \epsilon \varphi_{i}-d A}=\frac{\lambda_{i}}{\tilde{d} \beta_{i}} \sinh \left(\beta_{i} \xi+\gamma_{i}\right)
$$

while (22) gives the constraint $\tilde{d} \sum_{i} \beta_{i}^{2}=-8(\tilde{d}+1) k^{2}+d(2(D-2)-d \tilde{d} N) \mu^{2}$. The solution has an outer event horizon at $r^{\tilde{d}}=k(i$.e. at $t=\infty)$. The requirement that all the dilatonic fields be finite at the horizon implies that the constants $\beta_{i}$ are all equal, $\beta_{i}=\beta=\mu d$, and $\mu=2 k \sqrt{(\tilde{d}+1) /(d(D-2))}$. The functions $A$ and $B$ in the metric (11) are then given by

$$
\begin{aligned}
e^{-2(D-2) A / \tilde{d}} & =e^{(2(D-2)-d \tilde{d} N) \mu \xi / \tilde{d}} \prod_{i=1}^{N}\left(\frac{\lambda_{i}}{\tilde{d} \beta} \sinh \left(\beta \xi+\gamma_{i}\right)\right) \\
e^{2(D-2) B / d} & =(\cosh (k \xi))^{-(D-2) /(d \tilde{d})} e^{(2(D-2)-d \tilde{d} N) \mu \xi / \tilde{d}} \prod_{i=1}^{N}\left(\frac{\lambda_{i}}{\tilde{d} \beta} \sinh \left(\beta \xi+\gamma_{i}\right)\right) .
\end{aligned}
$$


The mass per unit $p$-volume is given by

$$
\begin{aligned}
m & =\frac{1}{4} \mu(2(D-2)-d \tilde{d} N)+\sum_{i} Q_{i} \cosh \gamma_{i} \\
& =\frac{1}{4} \mu(2(D-2)-d \tilde{d} N)+\sum_{i} \sqrt{Q_{i}^{2}+\frac{1}{16} \mu^{2} d^{2} \tilde{d}^{2}}
\end{aligned}
$$

where the charges $Q_{i}=\frac{1}{4} \lambda_{i}$, and we have chosen $\sinh \gamma_{i}=\tilde{d} \beta / \lambda_{i}$ so that the metric is Minkowskian at infinity.

\section{General dyonic solutions}

In dimension $D=2 n$, the field strength $F_{n}$ can carry both electric and magnetic charges. There are two types of dyonic $p$-brane solutions. In the first type, which can arise only when a $p$-brane solution involves more than one field strength, each field strength carries either electric or magnetic charge, but not both. The discussion of this type of dyonic solution is identical to that for purely electric or purely magnetic solutions, in terms of appropriatelydualised field strengths. In the second type of dyonic solution, each field strength carries both electric and magnetic charges. In this section, we shall discuss general single-scalar dyonic solutions of this second type. The relevant part of the bosonic Lagrangian is given by (2). After substituting the ansätze, the equations of motion give

$$
\begin{aligned}
& \phi^{\prime \prime}+X^{\prime} \phi^{\prime}=\frac{a}{2(n-1)^{2}}\left(\lambda_{1}^{2} e^{a \phi}-\lambda_{2}^{2} e^{-a \phi}\right) e^{2(n-1) A-2 X}, \\
& A^{\prime \prime}+X^{\prime} A^{\prime}=\frac{1}{4(n-1)^{2}}\left(\lambda_{1}^{2} e^{a \phi}+\lambda_{2}^{2} e^{-a \phi}\right) e^{2(n-1) A-2 X}, \\
& 2(n-1)^{2} A^{\prime 2}+\frac{1}{2}(n-1) \phi^{\prime 2}-n\left(X^{\prime 2}-\frac{2}{\rho} X^{\prime}\right)=\frac{1}{2(n-1)}\left(\lambda_{1}^{2} e^{a \phi}+\lambda_{2}^{2} e^{-a \phi}\right) e^{2(n-1) A-2 X},
\end{aligned}
$$

where $X=(n-1)(A+B)$ and satisfies (6). The parameters $\lambda_{1}$ and $\lambda_{2}$ are associated with the magnetic and electric charges of the solution. Defining new functions $q_{1}$ and $q_{2}$ by

$$
A=\frac{1}{4(n-1)}\left(q_{1}+q_{2}-2 \log \frac{\lambda_{1} \lambda_{2}}{n-1}\right), \quad \phi=\frac{a}{2(n-1)}\left(q_{1}-q_{2}\right)+\frac{1}{a} \log \frac{\lambda_{2}}{\lambda_{1}},
$$

the equations of motion (27) become, in terms of the new radial variable $\xi$ defined in (9),

$$
\begin{aligned}
& \ddot{q}_{1}=e^{\alpha q_{1}+(1-\alpha) q_{2}}, \quad \ddot{q}_{2}=e^{(1-\alpha) q_{1}+\alpha q_{2}}, \\
& H \equiv \frac{\alpha}{2(2 \alpha-1)}\left(p_{1}^{2}+p_{2}^{2}\right)+\frac{\alpha-1}{2 \alpha-1} p_{1} p_{2}-e^{\alpha q_{1}-(1-\alpha) q_{2}}-e^{(1-\alpha) q_{1}+\alpha q_{2}}=8 n k^{2}
\end{aligned}
$$

where

$$
\alpha \equiv \frac{1}{2}+\frac{a^{2}}{2(n-1)}=\frac{\Delta}{2(n-1)},
$$


and $H=H\left(p_{1}, p_{2}, q_{1}, q_{2}\right)$ is the Hamiltonian. Thus Hamilton's equations $\dot{q}_{i}=\partial H / \partial p_{i}$ imply that

$$
p_{1}=\alpha \dot{q}_{1}+(1-\alpha) \dot{q}_{2}, \quad p_{2}=(1-\alpha) \dot{q}_{1}+\alpha \dot{q}_{2}
$$

while $\dot{p}_{i}=-\partial H / \partial q_{i}$ gives precisely the equations of motion (29).

As far as we know, the equations (29), which have the general form of Toda equations, cannot be solved completely and explicitly for general values of $\alpha . \rrbracket$ There are, however, two values of $\alpha$ for which the equations do become completely solvable, namely

$$
\begin{array}{lll}
\alpha=1: & \ddot{q}_{1}=e^{q_{1}}, & \ddot{q}_{2}=e^{q_{2}}, \\
\alpha=2: & \ddot{q}_{1}=e^{2 q_{1}-q_{2}}, & \ddot{q}_{2}=e^{2 q_{2}-q_{1}} .
\end{array}
$$

The first case gives two independent Liouville equations, and the second case gives the $S U(3)$ Toda equations. The solutions are

$$
\begin{array}{ll}
\alpha=1: & \left.\left.e^{-\frac{q_{1}}{2}}=\frac{1}{\sqrt{2} \beta_{1}} \sinh \left[\beta_{1} \xi+\alpha_{1}\right)\right], \quad e^{-\frac{q_{2}}{2}}=\frac{1}{\sqrt{2} \beta_{2}} \sinh \left[\beta_{2} \xi+\alpha_{2}\right)\right], \\
\alpha=2: & e^{-q_{1}}=\sum_{i=1}^{3} f_{i} e^{\mu_{i} \xi}, \quad e^{-q_{2}}=\sum_{i=1}^{3} g_{i} e^{-\mu_{i} \xi}
\end{array}
$$

where $\beta_{i}$ and $\alpha_{i}$ are arbitrary constants of integration. In the second case, the constants $\mu_{i}, f_{i}$ and $g_{i}$ satisfy the relations

$$
\begin{array}{r}
\mu_{1}+\mu_{2}+\mu_{3}=0, \\
f_{1} f_{2} f_{3}\left(\mu_{1}-\mu_{2}\right)^{2}\left(\mu_{2}-\mu_{3}\right)^{2}\left(\mu_{3}-\mu_{1}\right)^{2}=-1, \\
g_{1}=-f_{2} f_{3}\left(\mu_{2}-\mu_{3}\right)^{2}, \quad \text { and cyclically . }
\end{array}
$$

The Hamiltonian is conserved, and in both cases the energy is equal to $8 n k^{2}$;

$$
\begin{array}{ll}
\alpha=1: & H=2\left(\beta_{1}^{2}+\beta_{2}^{2}\right)=8 n k^{2}, \\
\alpha=2: & H=\mu_{1}^{2}+\mu_{2}^{2}+\mu_{1} \mu_{2}=8 n k^{2} .
\end{array}
$$

Let us discuss the two cases in more detail. When $\alpha=1$, we have $a^{2}=n-1$ and hence $\Delta=2(n-1)$. In supergravity theories, the dilaton prefactors for all field strengths satisfy the condition $\Delta \leq 4$ [10], and hence $\alpha=1$ dyonic solutions can only arise for 3 -form field

\footnotetext{
${ }^{1}$ For all values of $\alpha$, there is special solution with $q_{1}=q_{2}$, and it follows from (28) that the dilaton field $\phi$ is constant, $a \phi=\log \left(\lambda_{1} / \lambda_{2}\right)$, and $A$ satisfies a Liouville equation, as in the case of purely electric or purely magnetic solutions. In the case of $a=0(\alpha=1 / 2)$, the dilaton field $\phi$ vanishes, and the effective total charge is given by $\lambda=\sqrt{\lambda_{1}^{2}+\lambda_{2}^{2}}$. The rest of the analysis is identical to the purely electric or purely magnetic solutions with charge $\lambda$.
} 
strengths in $D=6$ and 2-form field strengths in $D=4$. In $D=6$, the solution describes a black dyonic string, whilst in $D=4$, it describes a non-extremal dyonic black hole. In terms of the function $A$ and dilaton $\phi$, the solution is given by

$$
\begin{aligned}
e^{\sqrt{n-1} \phi-(n-1) A} & =\frac{\lambda_{1}}{\beta_{1} \sqrt{2(n-1)}} \sinh \left(\beta_{1} \xi+\alpha_{1}\right), \\
e^{-\sqrt{n-1} \phi-(n-1) A} & =\frac{\lambda_{2}}{\beta_{2} \sqrt{2(n-1)}} \sinh \left(\beta_{2} \xi+\alpha_{2}\right),
\end{aligned}
$$

with the constraint $\beta_{1}^{2}+\beta_{2}^{2}=4 n k^{2}$. The solution has an outer event horizon at $\rho=1 / k$ (i.e. at $\xi=\infty)$. As in the previous sections we should require that the curvature and the dilaton field $\phi$ be finite at this horizon, which implies that $\beta_{1}=\beta_{2} \equiv \beta=k \sqrt{2 n}$. The mass per unit $p$-volume and the electric and magnetic charges are given by

$$
\begin{aligned}
Q_{e} & =\frac{1}{4} \lambda_{2}, \quad Q_{m}=\frac{1}{4} \lambda_{1}, \\
m & =\sqrt{\frac{2}{n-1}}\left(Q_{m} \cosh \alpha_{1}+Q_{e} \cosh \alpha_{2}\right) \\
& =\sqrt{\frac{2}{n-1}}\left(\sqrt{Q_{m}^{2}+\frac{1}{4} n(n-1) k^{2}}+\sqrt{Q_{e}^{2}+\frac{1}{4} n(n-1) k^{2}}\right),
\end{aligned}
$$

where we have chosen $\sinh \alpha_{i}=\sqrt{2(n-1)} \beta / \lambda_{i}$ so that the metric approaches Minkowski spacetime asymptotically, and the dilaton vanishes at infinity. The usual extremal dyonic solutions [10] are recovered in the limit $k=0$. It is the supersymmetric dyonic string in $D=6$ with $\Delta=4$, or the non-supersymmetric dyonic black hole in $D=4$ with $\Delta=2$.

For $\alpha=2$, we have $a^{2}=3(n-1)$, and hence $\Delta=4(n-1)$. Thus the solution exists only in supergravity theories in $D=2 n=4$. Since in this case $\Delta=4$, there is only one participating 2-form field strength. The non-extremal black hole is given by (34) and (36). There are in total four independent parameters, and the solution of the $S U(3)$ Toda equations (33) gives

$$
\begin{aligned}
\frac{n-1}{\lambda_{1}^{4 / 3} \lambda_{2}^{2 / 3}} e^{-\frac{1}{3} a \phi-2(n-1) A} & =e^{-q_{1}}=\frac{c_{1} e^{\mu_{1} \xi}}{\nu_{1}\left(\nu_{1}-\nu_{2}\right)}-\frac{c_{2} e^{\mu_{2} \xi}}{\nu_{2}\left(\nu_{1}-\nu_{2}\right)}+\frac{e^{-\mu_{1} \xi-\mu_{2} \xi}}{c_{1} c_{2} \nu_{1} \nu_{2}} \\
\frac{n-1}{\lambda_{2}^{4 / 3} \lambda_{1}^{2 / 3}} e^{\frac{1}{3} a \phi-2(n-1) A} & =e^{-q_{2}}=\frac{e^{-\mu_{1} \xi}}{c_{1} \nu_{1}\left(\nu_{1}-\nu_{2}\right)}-\frac{e^{-\mu_{2} \xi}}{c_{2} \nu_{2}\left(\nu_{1}-\nu_{2}\right)}+\frac{c_{1} c_{2} e^{\mu_{1} \xi+\mu_{2} \xi}}{\nu_{1} \nu_{2}}
\end{aligned}
$$

where $\nu_{1}=2 \mu_{1}+\mu_{2}$ and $\nu_{2}=2 \mu_{2}+\mu_{1}$, together with the constraint $H=\mu_{1}^{2}+\mu_{2}^{2}+\mu_{1} \mu_{2}=$ $8 n k^{2}$. The solution has an outer horizon at $\rho=1 / k,($ i.e. at $\xi=\infty$,$) if the constants c_{1}$ and $c_{2}$ are both non-negative. As in the previous cases, we require that the dilaton field be finite at the horizon. No generality is lost in satisfying this condition by taking $\mu_{2}=0$. It follows from (36) that $\mu_{1}^{2}=8 n k^{2}$. Thus the $a=\sqrt{3}$ dyonic solution in $D=4$ is given by

$$
e^{-\phi / \sqrt{3}-2 A}=\frac{\lambda_{1}^{4 / 3} \lambda_{2}^{2 / 3}}{32 k^{2}}\left(c_{1} e^{4 k \xi}+\frac{1}{c_{1} c_{2}} e^{-4 k \xi}-2 c_{2}\right),
$$




$$
e^{\phi / \sqrt{3}-2 A}=\frac{\lambda_{2}^{4 / 3} \lambda_{1}^{2 / 3}}{32 k^{2}}\left(c_{1} c_{2} e^{4 k \xi}+\frac{1}{c_{1}} e^{-4 k \xi}-\frac{2}{c_{2}}\right) .
$$

If we require that $\phi, A$ and $B$ vanish at infinity, the coefficients $c_{i}$ are related to the charges $\lambda_{i}$ in the following way:

$$
c_{1}-2 c_{2}+\frac{1}{c_{1} c_{2}}=\sigma_{1} \equiv 32 k^{2} \lambda_{1}^{-4 / 3} \lambda_{2}^{-2 / 3}, \quad \frac{1}{c_{1}}-\frac{2}{c_{2}}+c_{1} c_{2}=\sigma_{2} \equiv 32 k^{2} \lambda_{2}^{-4 / 3} \lambda_{1}^{-2 / 3} .
$$

The dyonic black hole carries an electric charge $Q_{e}=\frac{1}{4} \lambda_{2}$ and a magnetic charge $Q_{m}=\frac{1}{4} \lambda_{1}$. The mass is given by

$$
m=\frac{k}{\sigma_{1}}\left(c_{1}-\frac{1}{c_{1} c_{2}}\right)+\frac{k}{\sigma_{2}}\left(c_{1} c_{2}-\frac{1}{c_{1}}\right) .
$$

The mass and the charges satisfy the Bogomol'nyi bound

$$
m^{2}-\left(Q_{e}^{2}+Q_{m}^{2}\right)=k^{2}\left(1+\frac{3}{\sigma_{1}}+\frac{6 c_{2}}{\sigma_{1}}+\frac{6}{\sigma_{2} c_{2}}\right) \geq 0
$$

where again $Q_{m}=\frac{1}{4} \lambda_{1}$ and $Q_{e}=\frac{1}{4} \lambda_{2}$. The solution becomes extremal if the parameter $k$ is set to zero. We shall study this limit in the following three special cases, namely $Q_{m}=0$, $Q_{e}=0$ or $Q_{m}=Q_{e}$. The first two cases can be obtained in the following limits: Defining $c_{i}=\alpha_{i} L$, we have

$$
\begin{aligned}
L \rightarrow 0: & Q_{m}=0, \quad Q_{e}=\frac{k \sqrt{2 \alpha_{1} \alpha_{2}}}{\alpha_{2}-2 \alpha_{1}}, \quad m=\frac{2 Q_{e}^{2}-2 k^{2}-k \sqrt{4 Q_{e}^{2}+k^{2}}}{k+\sqrt{4 Q_{e}^{2}+k^{2}}}, \\
L \rightarrow \infty: & Q_{e}=0, \quad Q_{m}=\frac{4 k \sqrt{2 \alpha_{1} \alpha_{2}}}{\alpha_{1}-2 \alpha_{2}}, \quad m=\frac{2 Q_{m}^{2}+2 k^{2}+2 k \sqrt{4 Q_{m}^{2}+k^{2}}}{k+\sqrt{4 Q_{m}^{2}+k^{2}}}
\end{aligned}
$$

In the extremal limit $k=0$ the mass is equal to $Q_{e}$ or $Q_{m}$ respectively, the Bogomol'nyi bound is saturated, and the solution becomes the usual supersymmetric purely electric or purely magnetic $a=\sqrt{3}$ black hole. In the third case $Q_{m}=Q_{e}=Q$, we have $c_{2}=1$, and the dilaton decouples. The mass is now given by

$$
m=\sqrt{8 Q^{2}+4 k^{2}}
$$

In the extremal limit $k=0$, the mass is equal to $2 \sqrt{2} Q=2 \sqrt{Q_{e}^{2}+Q_{m}^{2}}$, and hence it follows from (43) that the solution is not supersymmetric. This is generically the case in the extremal limit, if both $Q_{e}$ and $Q_{m}$ are non-zero. It is not clear whether they will survive quantum corrections.

\section{Conclusions}

In this paper, we have shown that the general equations of motion for isotropic single-scalar, multi-scalar and dyonic $p$-branes can be cast in the form of Liouville, Toda or Toda-like 
equations. Thus we have been able to construct the most general possible isotropic $p$-brane solutions for the Liouville and Toda cases. The solutions are generically non-extremal, and the dilatonic scalar fields are finite at the outer event horizon for appropriate choices of the constants of integration. In a certain limit, the solutions reduce to the previously-known extremal $p$-branes. In contrast to the usual black $p$-branes with $p \geq 1$, the non-extremal solutions that we obtained in this paper preserve the Poincaré invariance of the $p$-brane world-volume. This difference arises because the usual black $p$-brane solitons have metrics taking the form 12

$$
d s^{2}=e^{2 A}\left(-e^{2 f} d t^{2}+d x^{i} d x^{i}\right)+e^{2 B}\left(e^{-2 f} d r^{2}+r^{2} d \Omega^{2}\right),
$$

where $A, B$ and $f$ are functions of $r$, and $d \Omega^{2}$ is the metric on the unit $(D-d-1)$-sphere. Although a redefinition of the radial coordinate $r$ permits the metric $e^{-2 f} d r^{2}+r^{2} d \Omega^{2}$ in the transverse space to be recast in the form $d y^{m} d y^{m}$ appearing in (伍), the $p$-brane world-volume

metric $-e^{2 f} d t^{2}+d x^{i} d x^{i}$ will never take the fully-isotropic form $d x^{\mu} d x^{\nu} \eta_{\mu \nu}=-d t^{2}+d x^{i} d x^{i}$ appearing in (1) unless $p=0$, in which case there are no spatial world-volume coordinates $x^{i}$. Thus the non-extremal $p=0$ solutions that we obtained in this paper contain the standard non-extremal black holes, whereas the non-extremal $p \geq 1$ solutions in this paper do not overlap with the standard blackened $p$-brane solutions [12].

\section{Acknowledgement}

We are grateful to M.J. Duff for discussions.

\section{References}

[1] A. Dabholkar, G.W. Gibbons, J.A. Harvey and F. Ruiz Ruiz, Superstrings and solitons, Nucl. Phys. B340 (1990) 33.

[2] A. Strominger, Heterotic solitons Nucl. Phys. B343 (1990) 167.

[3] M.J. Duff and K.S. Stelle, Multi-membrane solutions of $D=11$ supergravity, Phys. Lett. B253 (1991) 113.

[4] M.J. Duff and J.X. Lu, Strings from fivebranes, Phys. Rev. Lett. 66 (1991) 1402.

[5] M.J. Duff and J.X. Lu, Elementary fivebrane solutions of $D=10$ supergravity, Nucl. Phys. B354 (1991) 141. 
[6] C.G. Callan, J.A. Harvey and A. Strominger, World-sheet approach to heterotic instantons and solitons, Nucl. Phys. B359 (1991) 611; World-brane actions for string solitons, Nucl. Phys. B367 (1991) 60.

[7] R. Güven, Black p-brane solutions of $D=11$ supergravity theory, Phys. Lett. B276 (1992) 49 .

[8] M.J. Duff, R.R. Khuri and J.X. Lu, String solitons, Phys. Rep. 259 (1995) 213.

[9] H. Lü, C.N. Pope, E. Sezgin and K.S. Stelle, Stainless super p-branes, Nucl. Phys. B456 (1995) 669.

[10] H. Lü and C.N. Pope, p-brane solitons in maximal supergravities, hep-th/9512012, to appear in Nucl. Phys. B.

[11] H. Lü and C.N. Pope, Multi-scalar p-brane solitons, hep-th/9512153, to appear in Int. J. Mod. Phys. A.

[12] M.J. Duff, H. Lü and C.N. Pope, The black branes of M-theory, preprint CTP-TAMU$14 / 96$. 